\title{
A Challenging Diagnosis of Leg Ulcer
}

\author{
Maria João Serpa, Susana Franco, Diana Repolho, Isabel Araújo, Sofia Mateus, António Martins Baptista, José Lomelino Araújo \\ Internal Medicine Department, Hospital Beatriz Ângelo, Lisbon, Portugal
}

Received: $17 / 08 / 2018$

Accepted: $17 / 09 / 2018$

Published: 04/10/2018

How to cite this article: Serpa MJ Franco S, Repolho D, Araújo I, Mateus S, Martins Baptista A, Lomelino Araújo J. A challenging diagnosis of leg ulcer. EJCRIM 2018;5: doi:10.12890/2018_000952.

Conflicts of Interests: The Authors declare that there are no competing interests.

This article is licensed under a Commons Attribution Non-Commercial 4.0 License

\section{ABSTRACT}

Ischaemic ulcers of the lower limb can have several aethiopathological mechanisms. For instance, the uncommon Martorell hypertensive leg ulcer is an infrequent complication of long-term uncontrolled arterial hypertension and is largely ignored and underdiagnosed. The typical ulcer is on the lower leg, is extremely painful and presents in female patients over 60 years of age with cardiovascular risk factors, mainly severe arterial hypertension. The diagnosis is histological, with obliteration of the arterioles by arteriolosclerosis. In these cases, antihypertensive medication is the standard treatment. We describe the case of an African-American female patient with long-term hypertension who presented with a very painful leg ulcer. The diagnosis was complicated by the coexistence of more common causes of ulcer. Despite analgesic medicines, pain relief was only obtained with control of hypertension. A definite diagnosis was made following biopsy.

In describing this diagnosis of an ischaemic leg ulcer, we highlight the need to consider the skin as a target organ of arterial hypertension.

\section{LEARNING POINTS}

- Martorell hypertensive leg ulcer is an underdiagnosed cutaneous lesion arising from long-term uncontrolled hypertension.

- The diagnosis is confirmed by histopathology.

- Standard treatment is adequate control of blood pressure.

\section{KEYWORDS}

Martorell ulcer, hypertensive ulcer, cutaneous pain

\section{CASE DESCRIPTION}

A 76-year-old black woman presented to the emergency department with excruciating pain in a supramalleolar cutaneous lesion on the medial aspect of her right leg, with a 10-day evolution, without fever. The cutaneous lesion was oval, about $5 \mathrm{~cm}$ in diameter, had an irregular outline with well-defined limits, was superficial with exudative, granulated and haemorrhagic areas, without devitalized tissue, and very painful, and was associated with surrounding cellulite of the leg to the knee (Figs. 1 and 2). Femoral pulses were palpable and symmetric, but distal pulses were absent in both legs. The pain was very intense (10/10) and sharp in the ulcer, with occasional shooting pain through the leg, worsened by touch and mobilization and slightly relieved by paracetamol.

The patient had a more than 20-year history of hypertension, with target organ damage: she had nephrosclerosis with chronic renal disease stage $3 b$ (GFR $40 \mathrm{ml} / \mathrm{min} / \mathrm{m}^{2}$ MDRD). She also had dyslipidaemia, had experienced an atheroembolic ischaemic stroke 13 years previously without sequelae, and had vertigo. 


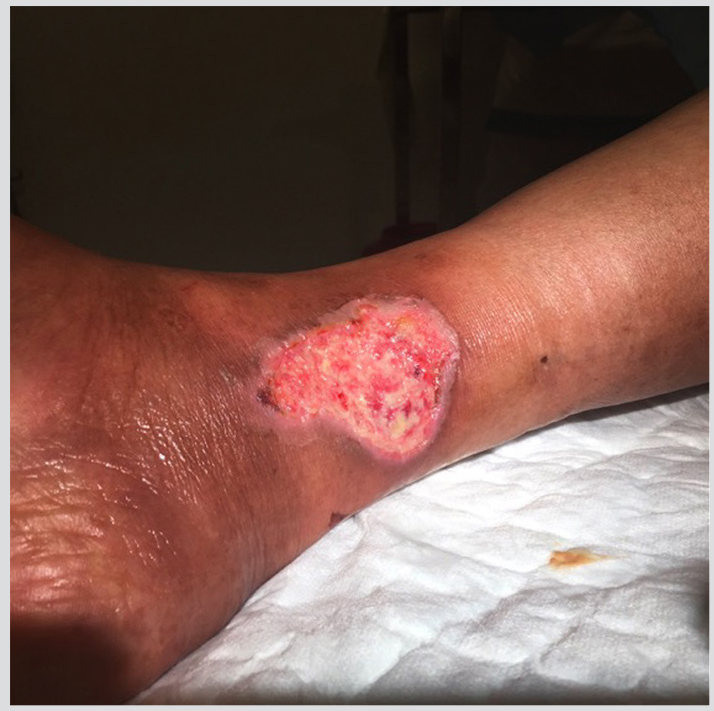

Figure 1. Medial supramalleolar ulcer

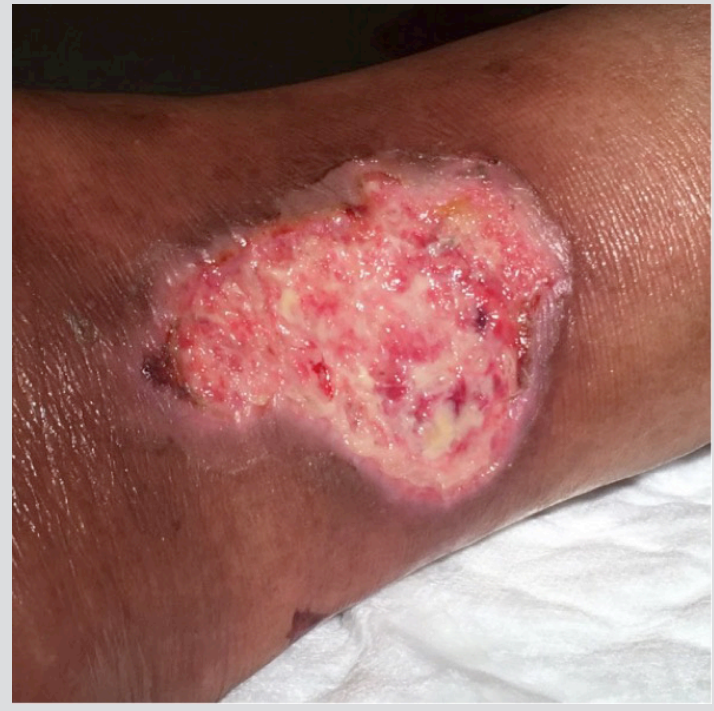

Figure 2. Close-up view of the ulcer

Laboratory results showed normochromic normocytic anaemia (haemoglobin $9.3 \mathrm{~g} / \mathrm{dl}$ ), without vitamin or iron deficiency, CPR $10 \mathrm{mg} / \mathrm{dl}$ without leucocytosis, mild renal failure with stable plasmatic creatinine of $1.97 \mathrm{mg} / \mathrm{dl}$, and normal calcium.

The patient was submitted to surgical debridement by the plastic surgery department and received 7 days of flucloxacillin. Arterial Doppler echography of the lower limbs performed by the vascular surgery department revealed obstructive arterial disease in the femoral, popliteal and distal regions with occlusion of the right femoral superficial artery and monophasic flow in the popliteal artery under the knee and distal arteries. The patient was proposed for elective arteriography.

When the patient was admitted to hospital, analgesic therapy was increased to opioids. As infection was suspected, a wide-spectrum antibiotic was prescribed despite negative blood cultures.

Hypertension was very difficult to control despite the use of five classes of drugs (diuretic, calcium channel blocker, beta-blocker, angiotensinconverting enzyme (ACE) inhibitors and clonidine), with occasional demand for IV nitrate. The patient developed bradycardia (45 bpm) and weakness as an adverse effect of clonidine and the drug had to be suspended. Her pain resolved only when adequate blood pressure control was achieved. The patient was weaned from opioids and discharged with only paracetamol and pregabalin as analgesics. The ulcer had healed completely 3 months later.

The lesion was biopsied and the diagnosis of Martorell hypertensive leg ulcer confirmed (Fig. 3). Adequate blood pressure control is key for treatment of this condition, as shown in our case.



Figure 3. Histological photomicrographs of the skin lesion, representative of Martorell hypertensive leg ulcer. Right panel: haematoxylin-eosin stained section showing epidermis ulceration, vessel vasculitis and dermal necrosis and haemorrhage (magnification $\times 2$ ). Left panel: haematoxylin-eosin stained section showing vessel detail with vasculitis, epidermal necrosis and haemorrhage (magnification $\times 20$ ). 


\section{DISCUSSION}

The diagnosis was not straightforward due to rarity of the aetiology and the fact that the patient also had peripheral obstructive arterial disease, which has been described in the literature in $45 \%$ of cases $^{[1]}$. The absence of typical symptomatology (intermittent claudication, relief of pain with rest, and changes in the colour (white) and temperature of the extremities (cold)), in addition to the ulcer itself, the gender (feminine) and age (above 60$)^{[2]}$ of the patient ${ }^{[3]}$ and, especially, resolution of the ulcer and pain only with adequate control of blood pressure, more or less excluded peripheral obstructive arterial disease as an aetiology and raised the suspicion of a cutaneous consequence of undercontrolled long-term hypertension. Pain is an important clinical sign in Martorell hypertensive leg ulcer ${ }^{[4]}$. The biopsy of the ulcer, with evidence of arteriolosclerosis and hyalinosis, confirmed the diagnosis of Martorell hypertensive leg ulcer ${ }^{[5]}$.

Despite the known effects of atherosclerosis of large and medium blood vessels (stroke, myocardial infarction) and microangiopathy of capillaries (collagen vascular diseases), arteriolosclerosis is underrated ${ }^{[1]}$. Arteriolosclerosis also has harmful effects on the heart, brain, kidneys and other organs ${ }^{[1]}$. The main mechanism in Martorell hypertensive leg ulcer is an arteriolosclerosis process involving the vessels of the dermis ${ }^{[2]}$.

The differential diagnosis includes vasculitis, calciphylaxis ${ }^{[4]}$, pyoderma gangrenosum ${ }^{[3]}$ and skin thrombosis (thrombophilia, antiphospholipid syndrome, cryoglobulinaemia) ${ }^{[2]}$.

Antihypertensive drugs are the mainstay of treatment of Martorell hypertensive leg ulcer ${ }^{[3]}$. Non-selective $\beta$-blocking agents are contraindicated in these patients due to low cardiac output and the resultant reduced skin perfusion pressure ${ }^{[3]}$. However, selective $\beta 1$ blocking agents such as bisoprolol, and ACE inhibitors such as lisinopril, do not have these disadvantages and can be prescribed safely in patients with this condition ${ }^{[3]}$.

Further treatment includes promotion of dermal blood flow, anticoagulants and local atraumatic debridement (surgical or vacuum-assisted closure $(\mathrm{VAC}))^{[3,4]}$, but all lack strong evidence of benefit. However, VAC has advantages over conventional therapy in wound healing, comfort, and duration of treatment and hospitalization ${ }^{[3]}$.

Preventive measure include avoidance of smoking and trauma, graft care using hydrocolloid foams, skin care and medical elastic compression stockings $(25-30 \mathrm{mmHg})^{[4]}$ if limb oedema is present ${ }^{[2]}$.

Oral analgesics or lumbar sympathectomy (to block vasospasm and reduce vascular resistance) may be considered for pain contro[ ${ }^{[3]}$. Narcotic analgesics should be administered together with pregabalin or tricyclic antidepressants, because of the neuropathic pain, which is a common component ${ }^{[2]}$.

\section{CONCLUSION}

This case involving an elderly patient with a multiorgan ischaemic and hypertensive background seemed to be similar to others seen in our clinical practice, but the intensity of the pain made it uncommon. The histological diagnosis of a very rare entity allowed us to heal the ulcer and treat the pain with only management of arterial hypertension.

\section{REFERENCES}

1. Hafner J, Nobbe S, Partsch H, Läuchli S, Mayer D, Amann-Vesti B, et al. Martorell hypertensive ischemic leg ulcer: a model of ischemic subcutaneous arteriolosclerosis. Arch Dermatol 2010;146:961-968.

2. Hafner J, Nobe S, Läuchli S, Kerl K, French L, Pelivani N, et al. Martorell hypertensive ischemic leg ulcer must not be confounded with pyoderma gangrenosum: management is totally different. Rev Vasc Med 2013;1:5-8.

3. Vuerstaek J, Reeder S, Henquet C, Neumann H. Arteriolosclerotic ulcer of Martorell. J Eur Acad Dermatol Venereol 2010;24:867-874.

4. Hafner J. Calciphylaxis and Martorell hypertensive ischemic leg ulcer: same pattern - one pathophysiology. Dermatology 2016;232:523-533.

5. Aurora P. Características de las úlceras de Martorell: una revisión de la literatura. Ucrea - Repositorio Abierto de la Universidad de Cantabria, http://hdl.handle. net/10902/11859. 